\title{
Cost-effectiveness of implementing routine hearing screening using a tablet audiometer for pediatric cystic fibrosis patients receiving high-dose IV aminoglycosides
}

\author{
Shirley P Huang, PharmD; Cameron J McKinzie, PharmD, BCPPS, BCPS, CPP; and Casey R Tak, PhD, MPH
}

\section{What is already known about this subject}

- Cystic fibrosis patients receiving highdose intravenous aminoglycosides are at risk of developing hearing loss.

- Patients with hearing loss experience a long-term effect on quality of life.

\author{
What this study adds \\ - This study models the clinical and \\ economic outcomes of a tablet-based \\ audiometer compared with current \\ screening practices. \\ - A tablet-based audiometer can increase \\ earlier detection of hearing loss and \\ is cost-effective in the short term \\ at a willingness-to-pay threshold of \\ $\$ 150,000$
}

\author{
Author affiliations \\ Shirley P Huang, PharmD, Division of \\ Pharmaceutical Outcomes and Policy, \\ University of North Carolina at Chapel Hill; \\ Cameron J McKinzie, PharmD, BCPPS, BCPS, \\ CPP, University of North Carolina Medical \\ Center, Chapel Hill; and Casey R Tak, PhD, \\ $\mathrm{MPH}$, Division of Pharmaceutical Outcomes \\ and Policy, University of North Carolina at \\ Chapel Hill and University of North Carolina \\ Health Sciences at MAHEC, Asheville. \\ AUTHOR CORRESPONDENCE: \\ Shirley P Huang, 828.250.3916; \\ sphuang2@unc.edu
}

J Manag Care Spec Pharm 2021;27(2):157-65

Copyright $(2021$, Academy of Managed Care Pharmacy. All rights reserved.

and probabilistic sensitivity analyses were conducted to evaluate model uncertainty.

RESULTS: In a hypothetical cohort of 100 patients, routine screening using a tablet audiometer increased promptly detected hearing loss by 8 patients. There was an incremental gain of 3.2 QALYs at an increased cost of $\$ 333,826$ compared with current screening practices. This resulted in an incremental cost-effectiveness ratio (ICER) of $\$ 103,771$ per QALY. In the 1-way sensitivity analysis, the ICER ranged between $\$ 64,345$ and $\$ 258,830$ per QALY. 
CONCLUSIONS: Using a tablet audiometer for routine hearing screening appears to be a cost-effective option at a $\$ 150,000$ per QALY willingness-to-pay threshold when only considering the immediate benefits gained. This analysis did not examine the long-term effects of early detection in language development for pediatric patients.

Cystic fibrosis (CF) is a genetic disorder of the cystic fibrosis transmembrane conductance regulator (CFTR) protein, which primarily affects the lungs and is characterized by chronic lung infections. Pulmonary exacerbations result in the deterioration of lung function and increased morbidity and mortality. ${ }^{1}$ Bacteria associated with pulmonary exacerbations include Pseudomonas aeruginosa, a Gramnegative bacterium, methicillin-resistant Staphylococcus aureus (MRSA), and nontuberculous mycobacteria. Once established in the airways, complete eradication of these organisms is unlikely. In 2018 , more than $20 \%$ of pediatric CF patients were treated with intravenous (IV) antibiotics for a pulmonary exacerbation. ${ }^{2}$ Aggressive treatment with high-dose IV aminoglycosides (AG) is often used to treat pulmonary exacerbations caused by P. aeruginosa and delay chronic infection and deterioration of lung function. ${ }^{3}$

Pediatric CF patients are at a high risk of AG toxicities due to altered membrane permeability and increased lean body mass, causing greater drug distribution throughout the body. ${ }^{4}$ Persistent use of high-dose IV AG therapies has been reported to cause ototoxicity in numerous studies, ranging from $8 \%$ to $47 \% .^{5-10}$ Hearing loss can significantly influence language and speech development in children, and earlier detection is needed to prevent long-term effects of ototoxicity. Nearly two thirds of children with permanent childhood hearing loss reported complaints of stuttering and inability to speak clearly. ${ }^{11}$ The younger a child is when he or she receives care for hearing loss, the greater effect that hearing loss interventions have on language outcomes. ${ }^{11,12}$

Although the American Speech-Language-Hearing Association and the American Academy of Audiology have recommended screening guidelines for ototoxic medications, adherence to monitoring during treatment varies between clinics. ${ }^{13,14}$ In a 2011 survey of U.S. CF accredited care centers, $39.4 \%$ of pediatric CF centers did not perform audiology screening for ototoxicity. ${ }^{15}$ Among the centers that perform screening, there may not be a standardized approach that ensures all at-risk patients are consistently screened. A recent study conducted at the Cystic Fibrosis Care Center at Children's Mercy Kansas City, found that a pharmacist-implemented ototoxicity monitoring protocol

\section{FIGURE 1 Markov Model Schematic}

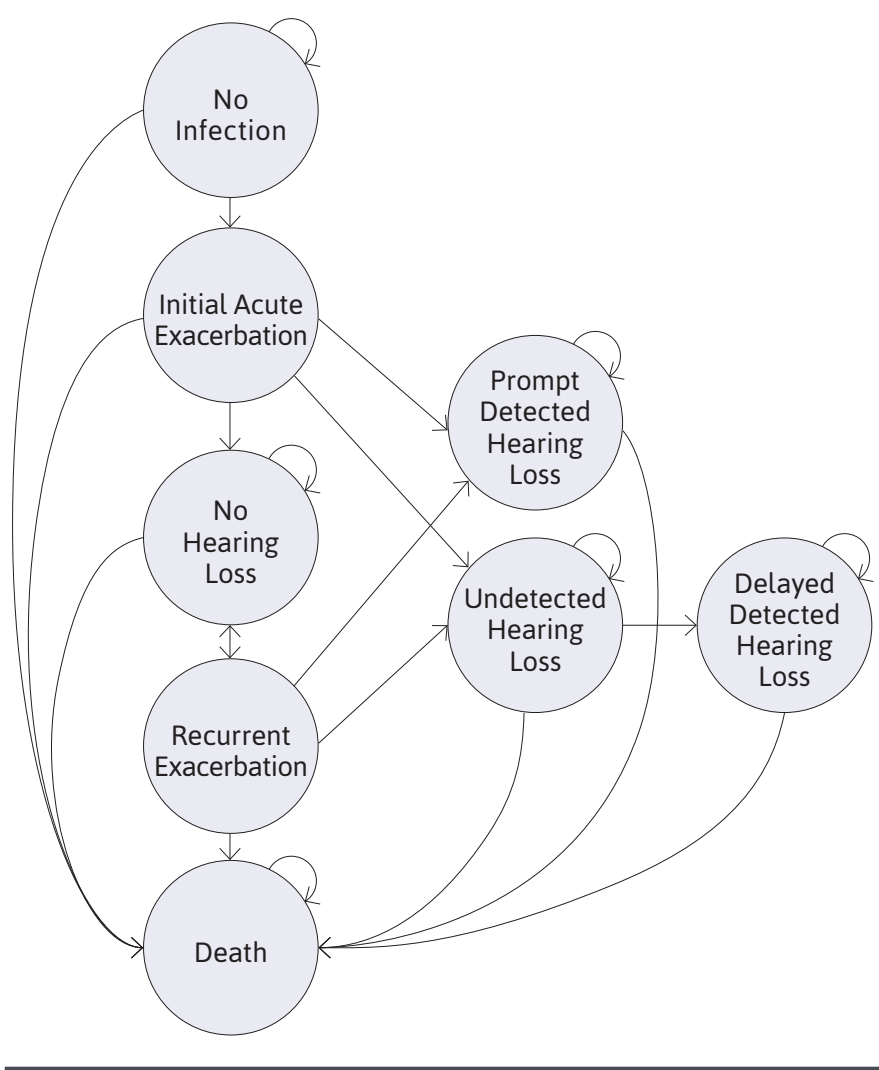

doubled the percentage of patients who received an audiogram after inhaled AG. ${ }^{16}$ While monitoring protocols help increase screening rates, there are still inherent limitations, since they rely on traditional screening practices.

The lack of screening in standard practice protocols may be attributed to the time-intensive nature of frequent testing needed to adequately detect early hearing loss. Current ototoxicity screening practices involve a referral to an audiology clinic where an audiologist would perform a pure-tone audiometry (PTA) test at baseline and posttreatment. ${ }^{13,14}$ There are numerous logistical barriers to implementing a monitoring program, as audiology services may not be located in each treatment center or sufficiently staffed to provide frequent ototoxicity monitoring. ${ }^{17}$ Due to the severity of $\mathrm{CF}$, patients manage a multitude of appointments, which can contribute to poor attendance to referrals. Furthermore, performing a PTA test can be burdensome and may not be necessary for all patients receiving high-dose IV AG. For these reasons, implementing a protocol using conventional screening methods may not be feasible. 


\section{TABLE 1 Markov Model Inputs}

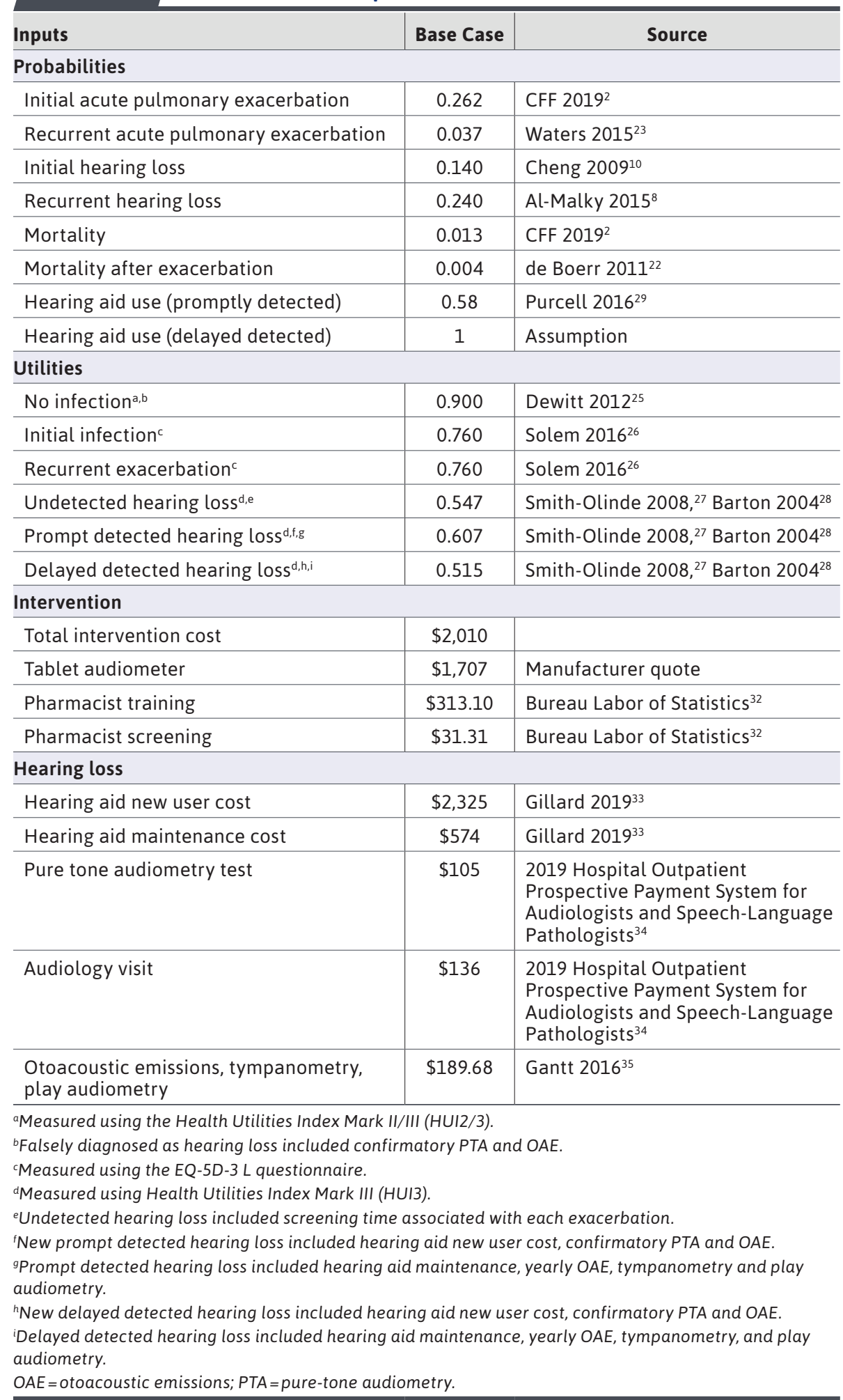

A tablet audiometer can be used to serve as a portable and accessible solution that can be used by health care professionals outside of the audiology department. ${ }^{18}$ A previous study on the benefits of tablet-based ototoxicity monitoring for routine screening in adult $\mathrm{CF}$ patients showed that it increased patient accessibility and broadened the range of personnel capable of administrating screening. ${ }^{19}$ Clinical pharmacists are uniquely positioned as the medication experts to conduct ototoxicity screening as a part of medication management and drug toxicity monitoring. Pharmacists play a pivotal role in minimizing the risk of treatment-induced adverse events..$^{20}$ In addition, pediatric $\mathrm{CF}$ patients followed by pharmacists in a therapeutic drug monitoring program achieved therapeutic drug targets more quickly. ${ }^{21}$

The purpose of this study was to assess the cost-effectiveness of a pharmacist-led routine audiometry screening for ototoxicity among pediatric patients using a clinically validated tablet audiometer.

\section{Methods}

\section{MODEL STRUCTURE}

A Markov decision-analytic model was constructed to compare the effect of routine tablet-based audiometry screening with current screening practices on costs and utilities from the societal perspective. The model had a time horizon of 3 years, with a cycle length of 1 month.

The Markov model included 8 health states as shown in Figure 1. A hypothetical cohort of 100 patients was used in this model to simulate the capacity of a pediatric CF center. A comprehensive literature review was conducted to determine screening probabilities, outcomes, and utilities associated with hearing loss (Table 1). 


\begin{tabular}{|c|c|c|c|c|c|c|}
\hline & \multicolumn{3}{|c|}{ Probability } & \multicolumn{3}{|c|}{ Incremental } \\
\hline & $\begin{array}{c}\text { Prompt } \\
\text { Detected HL }\end{array}$ & $\begin{array}{c}\text { Undetected } \\
\text { HL }\end{array}$ & $\begin{array}{c}\text { Delayed } \\
\text { Detected HL }\end{array}$ & QALY & Costs & ICER \\
\hline tervention & $11.70 \%$ & $0.69 \%$ & $0.08 \%$ & 3.22 & $\$ 333,827$ & $\$ 103,770$ \\
\hline CSP & $3.60 \%$ & $8.56 \%$ & $0.35 \%$ & & & \\
\hline
\end{tabular}

$\mathrm{CSP}=$ current screening practices; $H L=$ hearing loss; $Q A L Y=$ quality-adjusted life-year; $I C E R=$ incremental cost-effectiveness ratio.

The primary outcome for this analysis was the incremental cost-effectiveness ratio (ICER). All analyses were performed using Microsoft Excel 2020 (Redmond, WA).

\section{PROBABILITIES}

All patients entering the model were assumed to not have had previous hearing loss or a previous bacterial lung infection. Only acute pulmonary exacerbations that required high-dose IV AG were considered in this model. The risk of death in patients with acute exacerbations was assumed to be higher than in patients who do not experience any acute exacerbations. ${ }^{22}$

After their initial exacerbation, patients either had promptly detected hearing loss, undetected hearing loss, or no hearing loss. Patients who did not experience hearing loss at the initial exacerbation were still at risk for future hearing loss after another acute recurrent exacerbation requiring high-dose IV AG. ${ }^{2}$ The risk of developing hearing loss after the second pulmonary exacerbation was assumed to be higher than in the initial exacerbation and remained constant in future cycles. ${ }^{23}$ Patients with undetected hearing loss were screened at future exacerbations and, if detected, were categorized as delayed detected hearing loss. Transition probabilities for initial hearing loss varied by the initial and recurrent exacerbation. Due to a large range in hearing loss percentages reported in the published literature, conservative inputs were chosen from studies with more frequently reported values, and a constant ototoxicity risk was assumed after the second exacerbation..$^{8,10}$

All patients in the intervention strategy who experienced an exacerbation received high-dose IV AG at screening from the pharmacist during routine medication monitoring. Screening occurred at treatment initiation and after completion of treatment. The intervention inputs used in this study were derived from the SHOEBOX tablet audiometer (Ottawa, Ontario, Canada). In the no-intervention strategy for current screening practices, only a portion of patients were screened at an audiology visit using PTA after an exacerbation based on current practices and estimated screening completion rates from the literature (base-case value of $29 \%)^{15,17}$ To account for the inconsistency of successful referrals, it was assumed that only $47 \%$ of patients in a center that conducts screening received baseline and follow-up screenings.

A larger range of current screening practice probabilities was tested in the sensitivity analysis. The PTA in the current screening practice model was assumed to have had $100 \%$ sensitivity and $100 \%$ specificity, since the tablet audiometry validation studies used PTA as the standard comparison. The sensitivity and the specificity from the validation studies were used to treatment initiation and subsequent calculate the true positive hearing loss patients and false positive patients. ${ }^{24}$ Screening frequencies of the intervention and the current screening practice model remained consistent throughout the time horizon.

\section{UTILITIES}

All utilities had a decrement to adjust for having $\mathrm{CF}$ and experiencing pulmonary exacerbations. ${ }^{25,26}$ The utility for undetected hearing loss was weighted for mild to moderate hearing loss using previously published literature, and no utility gained from using a hearing aid. ${ }^{27,28}$ Detected hearing loss was weighted for mild to moderate hearing loss, with $58 \%$ receiving additional utility from a hearing aid that was calculated from the literature. ${ }^{27,29}$ Delayed detected hearing loss was weighted for moderate to severe hearing loss, and all patients had additional utility from a hearing aid.

\section{COSTS}

Intervention costs consisted of a fixed yearly cost of the tablet audiometer (which includes software license, tablet, and headphones); pharmacist training time; and pharmacist time spent administering the intervention. For this model, it was assumed that 5 pharmacists were trained to use the tablet audiometer (1 hour per pharmacist), and each screening took 30 minutes to complete. Screening time included testing at treatment initiation and after completion of therapy. Each exacerbation event included the episode cost of the exacerbation, as well as the additional screening time by the pharmacist. All patients in the intervention group after their first exacerbation incurred weighted costs for screening time associated with future exacerbations.

New prompt or new delayed detected hearing loss costs included a confirmatory PTA test and an otoacoustic emissions (OAE) test at an 


\section{FIGURE 2 Tornado Diagram for 1-Way Sensitivity Analysis}

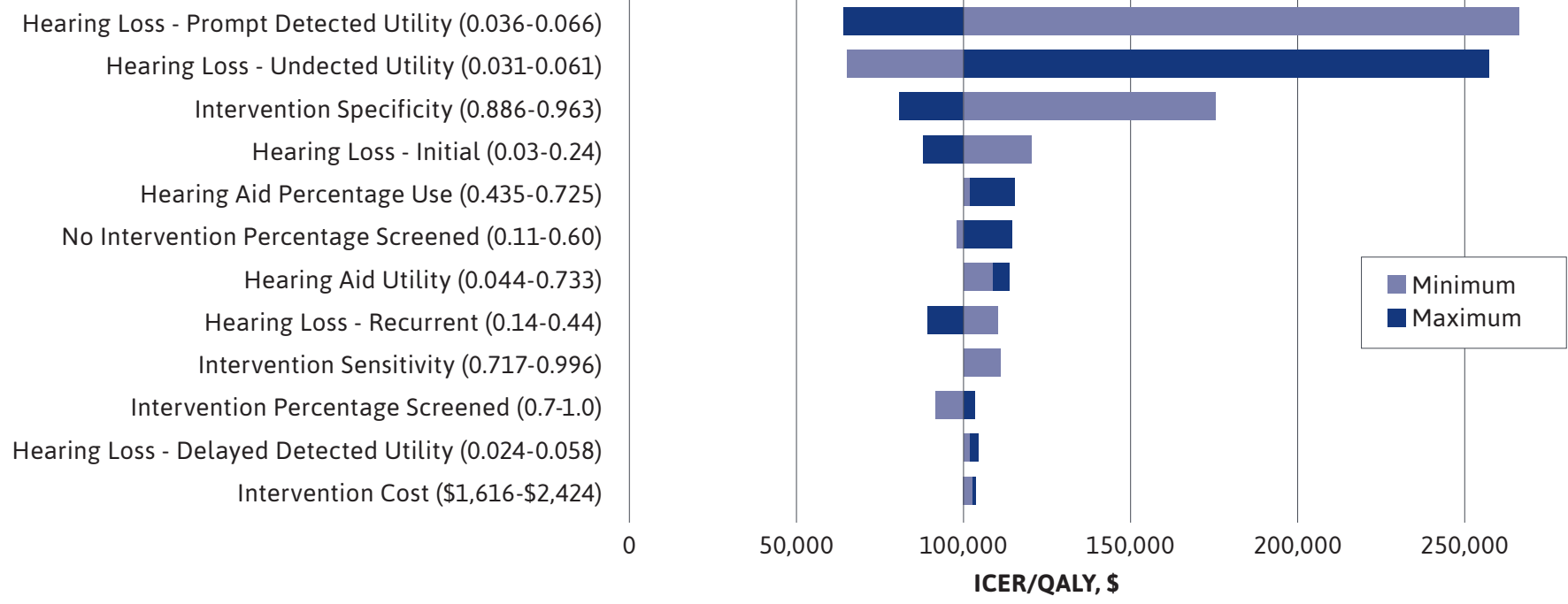

ICER = incremental cost-effectiveness ratio; $Q A L Y=$ quality-adjusted life-year.

audiology visit, as well as new hearing aid user costs. For the intervention and current screening practice model, it was assumed that $58 \%$ of patients in the prompt detected hearing loss required a hearing aid, whereas 100\% of patients with delayed detected hearing loss would require a hearing aid. Recurring costs for prompt or delayed detected hearing loss included hearing aid user maintenance, a yearly OAE test, tympanometry, and play audiometry. Patients who were falsely diagnosed as having hearing loss using the tablet audiometer would only incur the cost of a PTA test and an audiology visit. These patients would then reenter the model in the no hearing loss state.

All costs were expressed in U.S. dollars and were adjusted to 2019 dollars using the Personal Consumption Expenditure Healthcare Index. An annual discount of $3 \%$ was applied to costs and utilities.

\section{SENSITIVITY ANALYSES}

We conducted 1-way sensitivity analyses to explore each individual parameter's uncertainty, including any relevant assumptions, and its effect on the model results. If available, the standard deviation or $95 \%$ confidence intervals from the base-case value were used as the minimum and maximum limits. Otherwise, the range for the parameter was chosen from the literature. To test for a variety of health care personnel administering the test, a range of health care salaries were considered. Two-way sensitivity analyses varied intervention specificity alongside undetected hearing loss utility to evaluate potential negative long-term effects on language development and quality of life. A probabilistic sensitivity analysis (PSA) with 1,000 Monte-Carlo simulations was performed to examine the combined uncertainty of the model inputs. For the PSA, beta and gamma distributions were used for the probabilities and costs, respectively.

\section{SCENARIO ANALYSES}

Because this model assumed that all patients had no previous infection, a separate analysis was completed using higher inputs to account for chronic high-dose IV AG users who were assumed to have higher probabilities for acute exacerbations and associated hearing loss. The costs and utilities were derived by reweighting the hearing loss states to include severe hearing loss.

\section{Results}

\section{BASE CASE}

In the hypothetical cohort of 100 patients, the incremental cost of the intervention group was \$333,827, with an associated increase in QALYs of 3.2. This resulted in an estimated ICER of $\$ 103,770$, which is under the willingness-to-pay threshold (WTP) of \$150,000 (Table 2). Overall, $12.4 \%$ of the population had acquired hearing loss from IV AG use. In the 


\section{FIGURE 3 Two-Way Sensitivity Analysis Between Undetected Hearing Loss and Intervention Specificity}

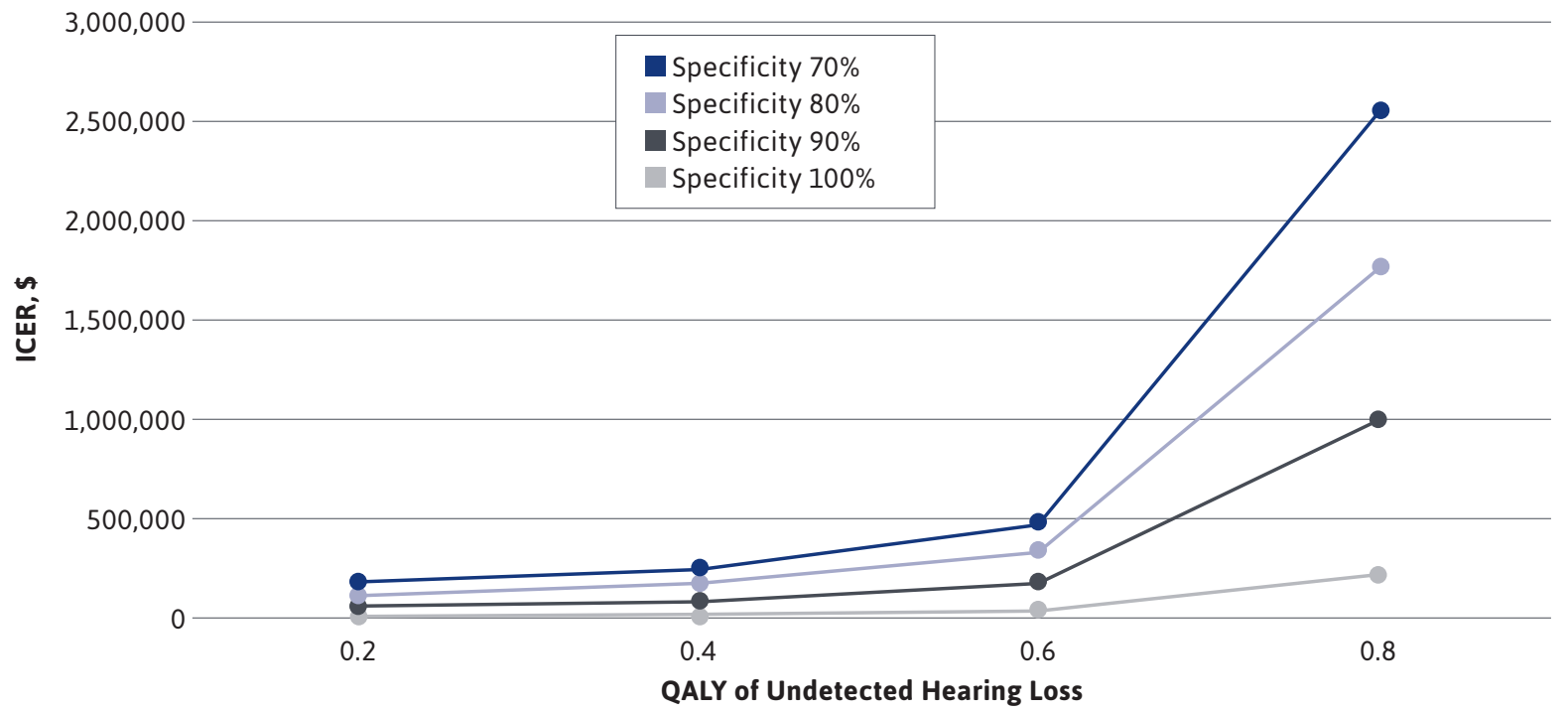

ICER = incremental cost-effectiveness ratio; QALY = quality-adjusted life-year.

intervention group, $11.5 \%$ were promptly detected, and $0.7 \%$ remained undetected. In the no intervention group with current screening practices, $3.5 \%$ were promptly detected, and $8.4 \%$ went undetected.

\section{SENSITIVITY AND SCENARIO ANALYSES}

Parameter estimates and assumptions were tested in the 1-way sensitivity analysis. The tornado diagram in Figure 2 reveals that the utility of promptly detected hearing loss, utility of undetected hearing loss, and specificity of the intervention had the greatest effect on the ICER and resulted in a potential non-cost-effective intervention at thresholds of $0.516,0.636$, and $90.7 \%$, respectively. The intervention cost had an insignificant effect on the ICER when a range of salaries were tested. In the 2-way sensitivity analysis, intervention specificity and undetected utility were varied. When undetected hearing loss utility was under 0.80 , the ranges of $70 \%-100 \%$ in intervention specificity resulted in ICERs below the WTP threshold (Figure 3). Inputs for the 1-way and 2-way sensitivity analyses are shown in Supplementary Table 1 and Supplementary Table 2 (available in online article).

The PSA results were plotted in a cost-effectiveness plane with WTP thresholds of $\$ 100,000$ and $\$ 150,000$ (Supplementary Figure 1, available in online article). In the 1,000 simulations, the mean ICER was $\$ 110,831$. The intervention was cost-effective $82.5 \%$ of the time at the $\$ 150,000$ threshold and $49.2 \%$ at the $\$ 100,000$ threshold.

In the chronic cohort scenario analysis, the ICER increased to $\$ 115,229$ (Supplementary Table 3, available in online article). This was primarily driven by increased exacerbations that increased screenings provided to each patient. With a larger proportion of patients receiving screening and acquiring hearing loss, detection would occur promptly, leading to increased costs of treatment and future audiology monitoring.

\section{Discussion}

Ototoxicity in $\mathrm{CF}$ patients has been increasingly reported in the literature. Numerous studies have recommended implementing targeted ototoxicity screening in standard practice. ${ }^{6}$ There is currently no established screening protocol that allows for a high completion rate in CF patients. There have been reports of implementing ototoxicity monitoring programs in various institutions for other disease states, but these efforts have been met with barriers due to time constraints of audiology departments and staffing limitations. ${ }^{17}$ Using a tablet audiometer can funnel patients with suspected hearing loss to audiology services and increase the efficiency of referrals. 
This was the first cost-effectiveness analysis of implementing a tablet audiometer to increase detection of ototoxicity. This model shows that routine screening using a tablet audiometer is a cost-effective option even when only considering the immediate utility gained. This analysis did not examine the long-term quality of life benefit of earlier detection in speech and language development. We suspect that the difference in QALYs of patients who are promptly detected and delayed detected will continue to increase as hearing loss symptoms worsen. Without timely screening, patients who self-report hearing loss symptoms will already have suffered extensive damage. In the United States, individuals with hearing loss symptoms report higher rates of unemployment and anxiety. ${ }^{30,31}$ The 2-way sensitivity analysis showed that, with increasing utility decrement of undetected hearing loss, the intervention will remain cost-effective even if accuracy decreases.

Currently, the model is assuming that the intervention is led by a group of pharmacists as they frequently interact with patients to assess the efficacy and adverse effects of drug therapy. Pharmacists were chosen because their unique training and education allows them to be aware of patients' exposure to medications, to consider their adverse effects, and to determine when to proactively trigger the need for screening. However, because resources and responsibilities of personnel vary between $\mathrm{CF}$ centers, it may not be feasible to require pharmacists for this intervention. Additional health care professionals could be trained to use the tablet audiometer if needed. The model was rerun with a range of other health care salaries, and there was only a slight decrease in the ICER, still resulting in a cost-effective solution.
A strength of this model is the distinction between the initial and recurrent exacerbation effects on probabilities, as well as the incorporation of specific hearing loss states. This increases differentiation in utilities and costs associated with each health state and the time hearing loss was detected. The model was also tested using a higher risk cohort of chronic high-dose IV AG users, which allows for better generalizability, since CF centers will have a mix of new and chronic users.

\section{LIMITATIONS}

There are, however, several limitations to our analysis. There were numerous studies that reported different incidences of acquired hearing loss associated with high-dose IV AG. In addition, there may be a nonlinear relationship between the number of high-dose IV AG courses and the risk of ototoxicity. Therefore, conservative inputs were chosen in the base-case analysis, and a chronic user cohort was evaluated for a higher risk population.

With limited literature on the utility of hearing loss, the different states of hearing loss were weighted using estimates on hearing aid use and associated utility. Utilities used in this model were measured using different instruments and may not be fully comparable. Utilities for the severity of hearing loss were also captured from caregivers and may not accurately represent the pediatric population.

This analysis focused on the population level and may not accurately describe how each individual patient experiences hearing loss symptoms and what costs they incur. Indirect and nonmedical costs, such as time missed from school and caretaker burden, were not included.

Finally, our time horizon does not fully capture the long-term costs and outcomes associated with ototoxicity and early or delayed hearing loss detection. Thus, the results described herein may have underestimated the cost-effectiveness of pharmacist-led audiology screening.

\section{Conclusions}

Hearing screenings using a tablet audiometer in pediatric patients with $\mathrm{CF}$ at risk for ototoxicity appears to be cost-effective from a societal perspective. Decision makers should be aware of the short-term and longterm effects of hearing loss and implement measures to ensure optimal outcomes. Future investigations are needed to explore the feasibility and implementation of pharmacist-led routine hearing screening using a tablet audiometer in pediatric and adult CF centers.

\section{DISCLOSURES}

Huang reports funding from the University of North Carolina and GlaxoSmithKline Health Outcomes Fellowship. GlaxoSmithKline had no involvement in the study creation, analysis, or manuscript composition. The other authors have nothing to disclose

\section{REFERENCES}

1. Waters V, Stanojevic S, Atenafu EG, et al. Effect of pulmonary exacerbations on long-term lung function decline in cystic fibrosis. Eur Respir J. 2012;40(1):61-66.

2. Cystic Fibrosis Foundation. 2018 Patient registry annual data report. 2019. Accessed December 30, 2020. https:// www.cff.org/Research/ResearcherResources/Patient-Registry/2018-PatientRegistry-Annual-Data-Report.pdf

3. Flume PA, Mogayzel PJ Jr, Robinson KA, et al. Cystic fibrosis pulmonary guidelines: treatment of pulmonary exacerbations. Am J Respir Crit Care Med. 2009;180(9):802-08. doi: 10.1164/ rccm.200812-1845PP. 
4. Molloy L, Nichols N. Infectious diseases pharmacotherapy for children with cystic fibrosis. J Pediatr Health Care. 2015;29(6):565-78.

5. Kreicher KL, Basuchard MJ, Clemmens CS, et al. Audiometric assessment of pediatric patients with cystic fibrosis. J Cyst Fibros. 2018;17(3):383-90.

6. Farzal Z, Kou Y, St. John R, et al. The role of routine hearing screening in children with cystic fibrosis on aminoglycosides: a systematic review. Laryngoscope. 2016;126(1):228-35.

7. Garinis AC, Cross CP, Srikanth P, et al. The cumulative effects of intravenous antibiotic treatments on hearing in patients with cystic fibrosis. J Cyst Fibros. 2017;16(3):401-09.

8. Al-Malky G, Dawson SJ, Sirimanna T, et al. High-frequency audiometry reveals high prevalence of aminoglycoside ototoxicity in children with cystic fibrosis. J Cyst Fibros. 2015;14(2):248-54.

9. Gleser MA, Zettner EM. Negative hearing effects of a single course of IV aminoglycoside therapy in cystic fibrosis patients. Int J Audiol. 2018;57(12):917-24.

10. Cheng AG, Johnston PR, Luz J, et al. Sensorineural hearing loss in patients with cystic fibrosis. Otolaryngol Head Neck Surg. 2009;141(1):86-90.

11. Ching TY, Dillon H, Button L et al. Age at intervention for permanent hearing loss and 5-year language outcomes. Pediatrics. 2017;140(3):e2016274.

12. Tomblin JB, Oleson JJ, Ambrose SE, et al. The influence of hearing aids on the speech and language development of children with hearing loss. Otolaryngol Head Neck Surg. 2014;140(5):403-09.

13. American Speech-Language-Hearing Association. Audiologic management of individuals receiving cochleotoxic drug therapy [guidelines]. 1994. Accessed January 7, 2021. https://www.asha.org/ policy/gl1994-00003/
14. American Academy of Audiology. Position statement and clinical practice guidelines: ototoxicity monitoring. October 2009. Accessed December 30, 2020. https://www.audiology.org/publications-resources/document-library/ ototoxicity-monitoring

15. Prescott WA Jr. National survey of extended-interval aminoglycoside dosing in pediatric cystic fibrosis pulmonary exacerbations. J Pediatr Pharmacol Ther. 2011;16(4):262-69.

16. Elson EC, Meier E, Oermann CM. The implementation of an aminoglycoside induced ototoxicity algorithm for people with cystic fibrosis. J Cyst Fibros. 2020;S1569-1993(20)30817-1. Online ahead of print. doi: 10.1016/j.jcf.2020.08.002

17. Konrad-Martin D, Poling GL, Garinis $A C$, et al. Applying U.S. national guidelines for ototoxicity monitoring in adult patients: perspectives on patient populations, service gaps, barriers and solutions. Int J Audiol. 2018;57(sup4):S3-S18.

18. Brungart D, Schurman J, Konrad Martin D, et al. Using tablet-based technology to deliver time-efficient ototoxicity monitoring. Int J Audiol. 2018;57(sup4):S25-S33.

19. Vijayasingam A, Frost E, Wilkins J, et al. Tablet and web-based audiometry to screen for hearing loss in adults with cystic fibrosis. Thorax. 2020;75(8):632-39.

20. Conway S, Balfour-Lynn IM,

De Ricke K, et al. European Cystic Fibrosis Society standards of care: framework for the Cystic Fibrosis Centre. J Cyst Fibros. 2014;13(Suppl 1):S3-S22.

21. Cies JJ, Varlotta L. Clinical pharmacist impact on care, length of stay, and cost in pediatric cystic fibrosis $(\mathrm{CF})$ patients. Pediatric Pulm. 2013;48(12):1190-94.

22. de Boer K, Vandemheen KL, Tullis E, et al. Exacerbation frequency and clinical outcomes in adult patients with cystic fibrosis. Thorax. 2011;66(8):680-85.

23. Waters V, Stanojevic S, Klingel M, et al. Prolongation of antibiotic treatment for cystic fibrosis pulmonary exacerbations. J Cyst Fibros. 2015;14(6):770-76.
24. Yeung J, Javidnia H, Heley S, et al. The new age of play audiometry: prospective validation testing of an iPad-based play audiometer. Otolaryngol Head Neck Surg. 2013;42(1):21.

25. DeWitt EM, Grussemeyer CA, Friedman JY, et al. Resource use, costs, and utility estimates for patients with cystic fibrosis with mild impairment in lung function: analysis of data collected alongside a 48-week multicenter clinical trial. Value Health. 2012;15(2):277-83.

26. Solem CT, Vera-Llonch M, Liu S, Botterman M, Castiglione B. Impact of pulmonary exacerbations and lung function on generic health-related quality of life in patients with cystic fibrosis. Health Qual Life Outomees. 2016;14:63.

27. Smith-Olinde L, Grosse SD, Olinde F, et al. Health state preferences scores for children with permanent childhood hearing loss: a comparative analysis of the QWB and HUI3. Qual Life Res. 2008;17(6):943-53.

28. Barton GR, Bankart J, David AC, et al. Comparing utility scores before and after hearing-aid provision: results according to the EQ-5D, HUI3 and SF-6D. Appl Health Econ Health Policy. 2004;3(2):103-05.

29. Purcell PL, Jones-Goodrich R, Wixneski $\mathrm{M}$, et al. Hearing devices for children with unilateral hearing loss: patient- and parent-reported perspectives. Int J Pediatr Otorhinolaryngol. 2016;90:43-48.

30. Petersen L, Roger C. Aminoglycosideinduced hearing deficits - a review of cochlear ototoxicity. S Afr Fam Pract. 2014;57(2):77-82.

31. Ruben RJ. Redefining the survival of the fittest: communication disorders in the 21st century. Laryngoscope. 2009;110(2):241-45.

32. U.S. Bureau of Labor Statistics. Occupational outlook handbook. Pharmacists. Retrieved November 28, 2019. Accessed December 30, 2020. https://www.bls.gov/ooh/healthcare/ pharmacists.htm 
33. Gillard DM, Harris JP. Cost-effectiveness of stapedectomy vs hearing aids in the treatment of otosclerosis. JAMA Otolaryngol Head Neck Surg. 2020;146(1):42-48.
34. American Speech-Language-Hearing Association. 2019 Hospital outpatient prospective payment system for audiologists and speech-language pathologists. November 12, 2018. Accessed December 30, 2020. https://www.asha.org/ uploadedFiles/2019-Hospital-OutpatientProspective-Payment-System-for-Audiologists-and-SLPs.pdf
35. Gannt S, Dionne F, Kozak FK, et al. Cost-effectiveness of universal and targeted newborn screening for congenital cytomegalovirus infection. JAMA Pediatr. 2016;170(12)1173-80.

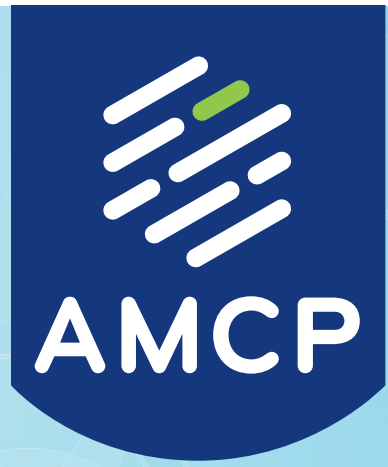

\section{DISEASE STATE Resource Center}

\section{Find in-depth information} and up-to-date resources about pressing disease states, including:

- COVID-19

- Heart failure

- And more

Learn more at www.amcp.org/diseasestates 